Acceso Abierto. Disponible en: https://revistas.up.ac.pa/index.php/faeco sapiens
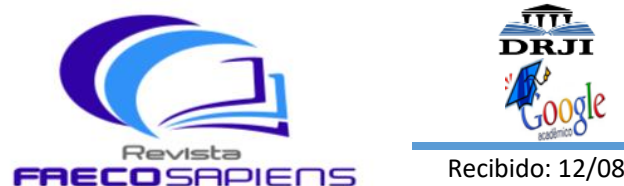

Volumen 4 - Número 1

enero-junio 2021

Recibido: $12 / 08 / 20$ Aceptado: 29/10/20 pp. 44-57

$\hat{\mathcal{A}}_{\text {meli.ca Indexada }}$

\title{
IMPORTANCIA DEL FLUJO DE EFECTIVO PROYECTADO COMO HERRAMIENTA DE CONTROL INTERNO PARA MANTENER EL NEGOCIO EN MARCHA EN LAS MICROS Y PEQUEÑAS EMPRESAS
}

Importance of the projected cash flow as a tool of internal control to keep the business running in micros and small enterprises

Yiros Dakay Vásquez

Universidad de Panamá, Facultad de Administración de Empresas y Contabilidad, Panamá Email: yiros.dvr@gmail.com (iD https://orcid.org/0000-0002-5998-1056

\section{Xiomara Castrellón Calderón}

Universidad de Panamá, Facultad de Administración de Empresas y Contabilidad, Panamá Email: xiocas2002@yahoo.com (iD) https://0000-0002-3913-2858

\section{Resumen}

En la actualidad las empresas presentan problemas en su gestión de negocio, no utilizan el Flujo de Efectivo Proyectado como una herramienta de control interno para una eficiente gestión de las finanzas, lo que provoca que las mismas queden rezagadas, para enfrentar las responsabilidades en su entorno de modelo de negocio, al no contar con herramientas adecuadas para una gestión eficiente no cuenta con provisión a corto y mediano plazo, como metas para el logro de objetivos puntuales, lo que incurre en no alcanzar los objetivos deseados, y en otros aspecto desmejorando la confianza con proveedores y acreedores, lo que conlleva a una mala imagen para las empresas.

Para lograr un eficiente control interno, las micros y pequeñas empresas deben contemplar las normas, políticas y procesos mínimos, para poder lograr los resultados esperados, para tal fin se enuncian una serie de cumplimiento a incorporar toda empresa para mejorar las falencias que tengan.

Palabras Clave: provisión, control interno, flujo de efectivo proyectado y gestión.

\begin{abstract}
Companies currently have problems in their business management, they do not use Projected Cash Flow as an internal control tool for efficient finance management, which causes them to lag behind, to face responsibilities in their business model environment, by not having adequate tools for efficient management has no short- and medium-term provision, such as goals for achieving one-off goals, which does not achieve the desired objectives, and otherwise improved trust with suppliers and creditors, leading to a bad image for companies.

To achieve efficient internal control, micro and small businesses must look at the minimum rules, policies and processes, in order to achieve the expected results, for this purpose a series of compliances are set out that must be incorporated by every company to improve the gaps they have.
\end{abstract}

Keywords: provision, internal control, projected cash flow and management. 
REVISTA FAECO SAPIENS

ISSN L 2644-3821

Acceso Abierto. Disponible en: https://revistas.up.ac.pa/index.php/faeco sapiens

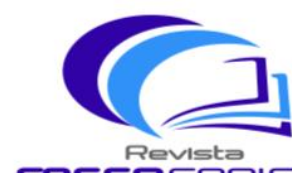

FคECロSคPIERS ताt Volumen 4 - Número 1

enero-junio 2021

Recibido: 12/08/20 Aceptado: 29/10/20 pp. 44-57

Indexada

\section{INTRODUCCIÓN}

En la actualidad las empresas presentan problemas en su gestión de negocio, no utilizan el Flujo de Efectivo Proyectado como una herramienta de control interno para una eficiente gestión de las finanzas, lo que provoca que las mismas queden rezagadas, para enfrentar las responsabilidades en su entorno de modelo de negocio, al no contar con herramientas adecuadas para una gestión eficiente no cuenta con provisión a corto y mediano plazo, como metas para el logro de objetivos puntuales, lo que incurre en no alcanzar los objetivos deseados, y en otros aspecto desmejorando la confianza con proveedores y acreedores, lo que conlleva a una mala imagen para las empresas.

Los nuevos emprendedores al crear empresas a nivel micro y pequeña, y de carácter familiar o entre amigos, carecen o desconocen la gestión de las finanzas y las ventaja que dan las mismas, para la planeación de los recurso y como hacerle frente a los compromisos, de tal manera que nuevos negocios por ser innovadores en sus inicios cumplen las expectativas y en ocasiones sobrepasándola, logrando rápidamente un alto nivel de ganancias, pero de la misma manera que llegan a ese pico, su descenso es inevitable, ya que no existen normas ni políticas de gestión del efectivo para compromisos y mantener el la marcha, en momentos cruciales que los el mercado y las tendencias varían.

Lo anterior expuesto nos da una serie de interrogantes como ¿Qué herramientas pueden usar para una eficiente gestión? ¿Cómo implementar esas herramientas? ¿Estas herramientas conllevan al logro de los objetivos? ¿Cumplen con las metas deseadas?

¿El flujo de efectivo proyectado, es una herramienta eficiente como control interno para lograr un resultado eficiente y de manera eficaz en la gestión del modelo de negocio para mantener las empresas en marcha? 
REVISTA FAECO SAPIENS

ISSN L 2644-3821

Acceso Abierto. Disponible en:

https://revistas.up.ac.pa/index.php/faeco sapiens
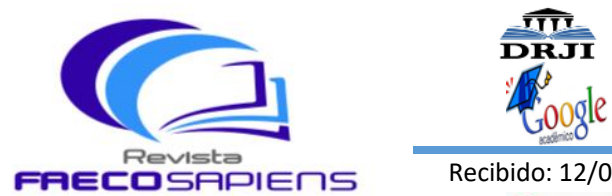

Volumen 4 - Número 1

enero-junio 2021

Recibido: $12 / 08 / 20$ Aceptado: $29 / 10 / 20$ pp. $44-57$

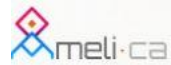

Indexada

\section{"IMPORTANCIA DEL FLUJO DE EFECTIVO PROYECTADO COMO HERRAMIENTA DE CONTROL INTERNO PARA MANTENER EL NEGOCIO EN MARCHA EN LAS MICROS Y PEQUEÑAS EMPRESAS"}

El presente estudio, pretende demostrar como las micro y pequeñas empresas en Panamá, han venido desarrollando problemas de gestión para enfrentar las responsabilidades en su entorno de modelo de negocio, al no contar con herramientas adecuadas para una gestión eficiente a corto y mediano plazo, no alcanzan los objetivos deseados, y en otros aspectos desmejorando la confianza con proveedores, acreedores entre otros, lo que conlleva a una mala imagen para las empresas. A la vez, demostrar que, utilizando el Flujo de Efectivo Proyectado como una herramienta para una gestión eficiente para mantener el negocio en marcha, aplicando el control interno bajo normas, políticas y procesos, puede mejorar la eficiencia en el cumplimiento, de dichas responsabilidades frente a tercero. Este estudio se basa en la descripción de hechos y experiencia obtenida por el investigador en el trascurrir del tiempo. El mismo, busca brindar a las micros y pequeñas empresas una herramienta eficiente y eficaz, la cual ayude a lograr los objetivos planteados.

Para Serrano (2016) disponer de una información contable relevante y fiable es más crítico en momentos de crisis económica, donde las empresas deben competir para acceder a los recursos financieros limitados disponibles en el mercado (p. 20).

Por la finalidad de este estudio, se trata de una investigación tipo descriptiva como expresa Lerma (2014, citado por Mera 2017) donde establece que: "Su objetivo es identificar el estado, la particularidad, factores y como son los procedimientos actuales en fenómenos y hechos que suelen ocurrir en forma completamente natural, sin explicar las vinculo que los identifiquen" (p. 36).

El objetivo de este tipo de investigación para Castillero (2020) es "únicamente establecer una descripción lo más completa posible de un fenómeno, situación o elemento concreto, sin buscar ni causas ni consecuencias de éste. Mide las características y observa la configuración y los procesos que componen los fenómenos, sin pararse a valorarlos" (párr. 7).

\section{Antecedentes del Estudio}

Rojas (2016) en su tesis de Maestría titulada: Interpretación del Estado de Flujo de Efectivo del Banco Central del Ecuador Durante Los Años 2010-2013 mediante Análisis de componentes principales, sustentada en la Universidad del Azuay Ecuador, cuyo objetivo general fue el Análisis Proyectivo del Estado de Flujo de Efectivo para poder sustentar una adecuada toma de decisiones en las actividades financieras, operacionales, administrativas y comerciales.

Todas las empresas independientemente de la actividad a que se dediquen, necesitan de información financiera confiable, que proporciona el estado de flujo de efectivo que determina la capacidad que tiene la institución para generar el efectivo, lo que le permitirá cumplir con sus obligaciones inmediatas y directas, para sus proyectos de inversión y expansión. Además el flujo permite hacer un análisis de cada una de las partidas que inciden en 


\section{REVISTA FAECO SAPIENS ISSN L 2644-3821}

Acceso Abierto. Disponible en: https://revistas.up.ac.pa/index.php/faeco sapiens
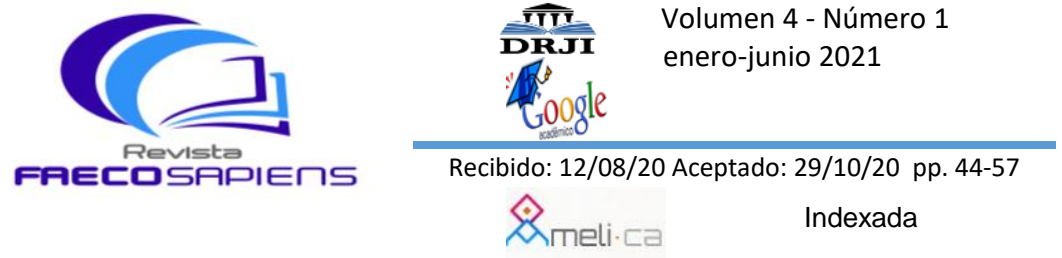

la generación de efectivo, el cual puede ser de gran utilidad para elaborar políticas y estrategias que permitan a la institución utilizar óptimamente sus recursos.

Al interpretar el estado de flujo de efectivo mediante componentes principales, se busca obtener información importante y surge como respuesta a la necesidad de determinar la salida de recursos en un momento determinado, como también un análisis proyectivo para sustentar la toma de decisiones en las actividades financieras, operacionales, administrativas y comerciales. (p. 13)

Zorrilla y Requena , en su tesis de titulada: El Estado de Flujos de Efectivo como Estrategia para la Eficiente Administración de los Fondos en las Empresas Comerciales de la Provincia de Huaura, cuyo objetivo general Demostrar la manera en que el Estado de Flujos de Efectivo como estrategia influye en la eficiente Administración de los Fondos de las Empresas Comerciales de la Provincia de Huaura, concluyo lo siguiente:

El Estado de Flujos de Efectivo como estrategia influye de manera significativa en la dinamización de la Administración de Fondos en las Empresas Comerciales de la Provincia de Huaura, por cuanto proporciona información muy importante para efectos de que las Gerencias de dichas Empresas puedan utilizarla en el complejo proceso de la toma de decisiones fundamentalmente orientadas a lograr una eficiente Administración de sus Fondos en lo que respecta a la recaudación, custodia y óptima utilización garantizando de esa manera el desarrollo de las actividades en un período determinado, basado en el conocimiento de los mecanismos que se han utilizado en el período anterior respecto a la generación de los flujos de fondos provenientes de las actividades de operación, inversión y financiamiento. ( 2015)

Claudia Toledo L. en su tesis Elaboración, Análisis e Interpretación del Estado de Flujos de Efectivo de una Droguería presenta que en la actualidad la globalización, la competitividad y demás factores de mercado requieren que las empresas dispongan de información financiera oportuna y certera para aprovechar oportunidades de negocios únicas.

Una herramienta financiera confiable lo constituye el Estado de Flujos de Efectivo cuyo fin es suministrar datos comprensibles y útiles, determinando el manejo y la habilidad de la empresa para generar flujos positivos de efectivo, en especial flujos provenientes del giro normal del negocio.

Las droguerías forman parte de un gran sistema de distribución de medicamentos y cuya importancia radica en lo económico y social, debido a que con sus productos brinda bienestar a la población, abastece al sector salubrista, genera empleo y contribuye con sus impuestos. En los últimos años las droguerías se han enfrentado a una competencia cada vez más fuerte dentro del gremio farmacéutico, por lo que se hace necesario contar con herramientas adecuadas para enfrentar los retos que conlleva el manejo de una empresa de este tipo. (Toledo López, 2016) 
REVISTA FAECO SAPIENS

ISSN L 2644-3821

Acceso Abierto. Disponible en:

https://revistas.up.ac.pa/index.php/faeco sapiens

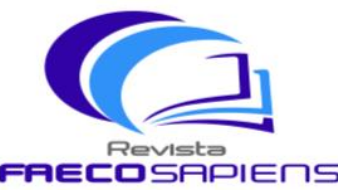

\&

cibido: $12 / 08 / 20$ Aceptado: 29/10/20 pp. $44-57$

Indexada

\section{Situación de las Micro y Pequeñas Empresas en Panamá}

La distribución de las micro y pequeñas a nivel de provincias y comarcas para el año 2016 según Autoridad de la Micro, Pequeña y Mediana Empresa (2017) refleja que "el sector está concentrado en la provincia de Panamá con el $45 \%$, seguido por la provincia de Chiriquí con el $13.7 \%$ y luego Panamá Oeste con el $8.2 \%$. Además, las que menos peso tiene son las comarcas con el 1\%, Darién con el 1.6\% y Bocas del Toro con el 2.4\%" ([AMPYME] p. 13).

Las micros y pequeñas empresas contaban con la mayoría de las empresas en las actividades de comercio al por menor con el $42.2 \%$ y $27.6 \%$ respectivamente; las medianas empresas en comercio al por mayor (incluye zonas francas) con el 26.2\%. En cuanto a las otras actividades con mayor cantidad de empresas por tamaño de empresa al 2016, se distinguen:

Micro: hoteles y restaurantes, y otras actividades de servicios,

Pequeña: comercio al por mayor y hoteles y restaurantes;

Tabla 1

Micro y Pequeñas Empresas en Panamá.

\begin{tabular}{|l|r|r|}
\hline \multicolumn{1}{|c|}{ Actividad Económica } & \multicolumn{2}{|c|}{2016} \\
\cline { 2 - 3 } & Micro & Pequeña \\
\hline Actividades administrativas y servicios de apoyo & 1,174 & 294 \\
\hline Actividades de organizaciones y órganos extraterritoriales & 4 & 1 \\
\hline Actividades financieras y de seguros & 799 & 250 \\
\hline Actividades inmobiliarias & 664 & 293 \\
\hline Actividades profesionales, científicas y técnicas & 1,636 & 559 \\
\hline Agricultura, ganadería, caza, silvicultura, pesca y actividades de serv. conexas & 43 & 19 \\
\hline Artes, entretenimiento y creatividad & 255 & 47 \\
\hline Comercio al por mayor (Incluye Zonas Francas) & 1,411 & 794 \\
\hline Comercio al por menor; reparación de los vehículos de motor y motocicletas & 18,272 & 1,844 \\
\hline Construcción & 369 & 271 \\
\hline Enseñanza & 585 & 183 \\
\hline Explotación de minas y canteras & 20 \\
\hline Hoteles y Restaurantes & 12 \\
\hline Industrias Manufactureras & 7,961 & 603 \\
\hline Información y comunicación & 3,257 & 597 \\
\hline Otras actividades de servicio & 177 & 113 \\
\hline Servicios sociales y relacionados con la Salud humana & 4,225 & 231 \\
\hline Suministro de agua; alcantarillado, gestión de desechos y activ de saneamiento & 1,501 \\
\hline Suministro de electricidad, gas, vapor y aire acondicionado & 31 & 196 \\
\hline Transporte, almacenamiento y correo & 8 \\
\hline Total & 9 & 5 \\
\hline
\end{tabular}

Nota: Realizado en base al Informe: “Evolución y Estado de las Mipyme y el Emprendimiento" (2017) 
REVISTA FAECO SAPIENS

ISSN L 2644-3821

Acceso Abierto. Disponible en:

https://revistas.up.ac.pa/index.php/faeco sapiens
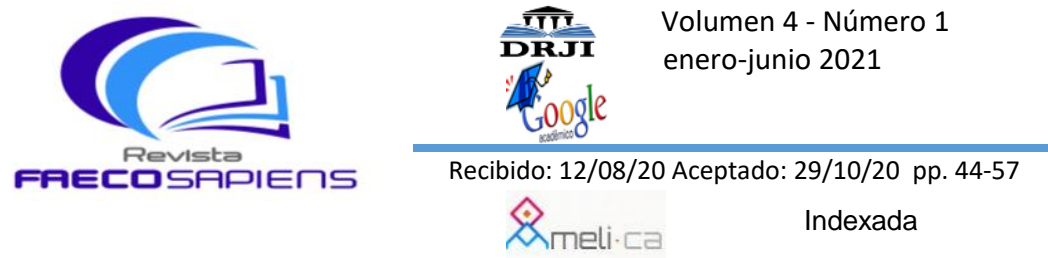

\section{Flujo de Efectivo Proyectado}

Una función de control efectivo debe considerar un plan de niveles de autoridad y responsabilidad, y separación de funciones claramente definidos con un sistema y procedimientos coherentes con las funciones, con el compromiso que se adopte una cultura de eficiencia y eficacia basada en controles

El control es efectivo al estar bien estructurado, es continuo y seguido por toda la organización. Debe estar alineado con las políticas que definen cómo hacer las actividades y con los propósitos de la empresa, participando, con las otras funciones de la administración, en la consecución cohesionada y coherente de los objetivos de hacer empresa, en la generación de confianza para la toma de las decisiones y en la calidad de desempeño de esta.

Como se menciona en la página Solo Contabilidad (2019) "El flujo de efectivo proyectado es una herramienta básica para la administración financiera, con ello se planifica el uso eficiente de efectivo, manteniendo saldos razonablemente cercanos a las permanentes necesidades de efectivo." (párr. 1)

En otras palabras, la proyección del efectivo es una herramienta indispensable en la gestión de las cuentas por pagar, paralela a las necesidades de la empresa, la cual ayuda a la gestión a provisionar la capacidad, para enfrentar responsabilidades y de esta manera poder mantener buenas relaciones comerciales con proveedores, a la vez que fortalece la imagen de la empresa y robustecer la confianza entre las partes.

Generalmente las salidas de efectivo son por pagos a proveedores, sueldos y salarios, gastos de fabricación desembolsables, gastos de administración y comercialización desembolsables, amortización de préstamos, inversiones en bienes de uso y otros. Por lo cual una buena gestión de basado en el flujo de efectivo como herramienta brindara la información oportuna y necesaria para que los administradores conozcan la situación de la empresa de antemano y puedan tomar las decisiones en el logro de los objetivos.

La información necesaria para realizar un flujo de efectivo requiere ser procesada en pasos que entre otros son los siguientes:

Saldo inicial de afectivo.

Ingresos proyectados por:

Ventas al contado.

Cobros a clientes por ventas a crédito.

Cobros

Desembolso por:

Pagos a proveedores

Gastos de Administración y venta.

Pagos de financiamientos 
REVISTA FAECO SAPIENS

\section{ISSN L 2644-3821}

Acceso Abierto. Disponible en:

https://revistas.up.ac.pa/index.php/faeco sapiens

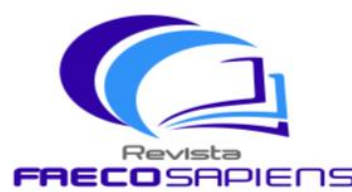

Volumen 4 - Número 1

enero-junio 2021

Recibido: $12 / 08 / 20$ Aceptado: 29/10/20 pp. 44-57

Indexada

La correcta realización del flujo de efectivo brinda la información de la liquidez que tiene la empresa, así, la toma de decisiones oportuna aporta una eficiente gestión para poder realizar adecuadamente sus actividades futuras.

Ahora, corresponde analizar cómo se gestiona el control interno a través de la adopción de esta herramienta en el área para enfrentar con éxito los riesgos. El proceso de control se aplica a todos los aspectos del desempeño y debe monitorearse y evaluarse en los niveles estratégico, administrativo y operativo. Lo que demostrará la efectividad de este.

La información que producen los sistemas y procesos de la función de control, respecto al desempeño esperado, pretende que los administradores tomar decisiones en la provisión y mitigación de los riesgos y la corrección de sus efectos. Las acciones y actividades de control basadas en políticas y plasmadas en procedimientos deben asegurar las actividades, dando respuesta a los riesgos en todos los niveles de la organización. Para que sean efectivas, las políticas deben estar escritas en manuales o directivas, e indicar qué se debe hacer. Por su parte, los procedimientos también, los manuales, deben indicar cómo se hace y vigilar su cumplimiento con el seguimiento de las actividades. Con respecto a ello, es preciso anotar que efectividad significa que los controles de la empresa son eficientes y eficaces con definido criterio de costo-beneficio, para lo cual la evaluación de estos debe estar acompañados, para poder ponderar y conocer su efectividad.

En consecuencia, el análisis del estado de flujo de efectivo es un tema muy importante ya que nos va a permitir una buena gestión de esta, así como la definición de la administración de la estructura del capital, la emisión de deuda, etc. Si llegamos a tener una buena administración del capital de trabajo nos va a permitir en el corto plazo la supervivencia de la empresa.

\section{Componentes del Flujo de Efectivo}

El flujo de efectivo es compuesto por cedulas que ayudan a determinar cada una de las gestiones por individual estableciendo los presupuestos para los objetivos de cada componente para poder realizar el flujo de efectivo proyectado pronosticando cada ingreso y egreso de los recursos necesarios para cumplir con el propósito del negocio.

En la figura 1. Componentes del Flujo de Efectivo, de detalla cómo está integrado, dicho flujo mostrando como cada componente esta interconectado en función de orden para poder lograr los objetivos deseado por la empresa, el primer la primera cedula a presupuestar es las ventas estimadas las que deben ser establecidas de acuerdo con los costos variables y fijos para conocer la cantidad mínima de venta donde la empresa este en punto equilibrio (no hay perdidas ni ganancias), de ahí se establece las cantidades que se esperar vender estimando la ganancia mensual por el periodo determinado, se continua con el proceso de comprar (costos variables) y las políticas de pago que se aceptarán, para mantener el inventario final para ventas, dando como resultado el presupuesto de costo de las ventas y por último el presupuesto de gasto operativos (costos fijos). 
REVISTA FAECO SAPIENS ISSN L 2644-3821

Acceso Abierto. Disponible en:

https://revistas.up.ac.pa/index.php/faeco sapiens

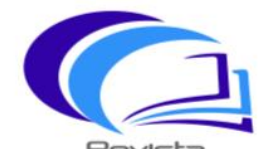

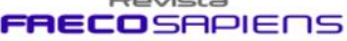

तार Volumen 4 - Número 1

DRJI enero-junio 2021

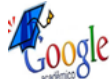

Recibido: $12 / 08 / 20$ Aceptado: 29/10/20 pp. 44-57 \&meli.raI Indexada 
Acceso Abierto. Disponible en:

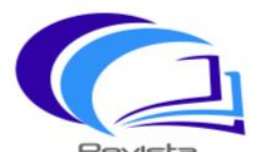

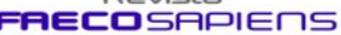
https://revistas.up.ac.pa/index.php/faeco sapiens

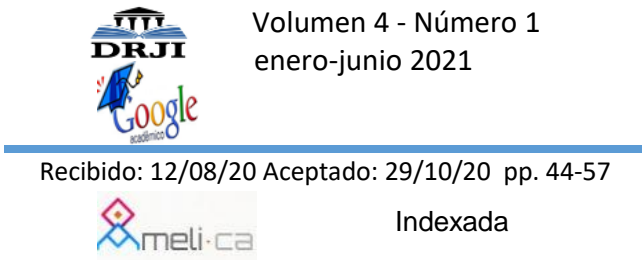

\section{Figura 1.}

Componentes del Flujo de Efectivo

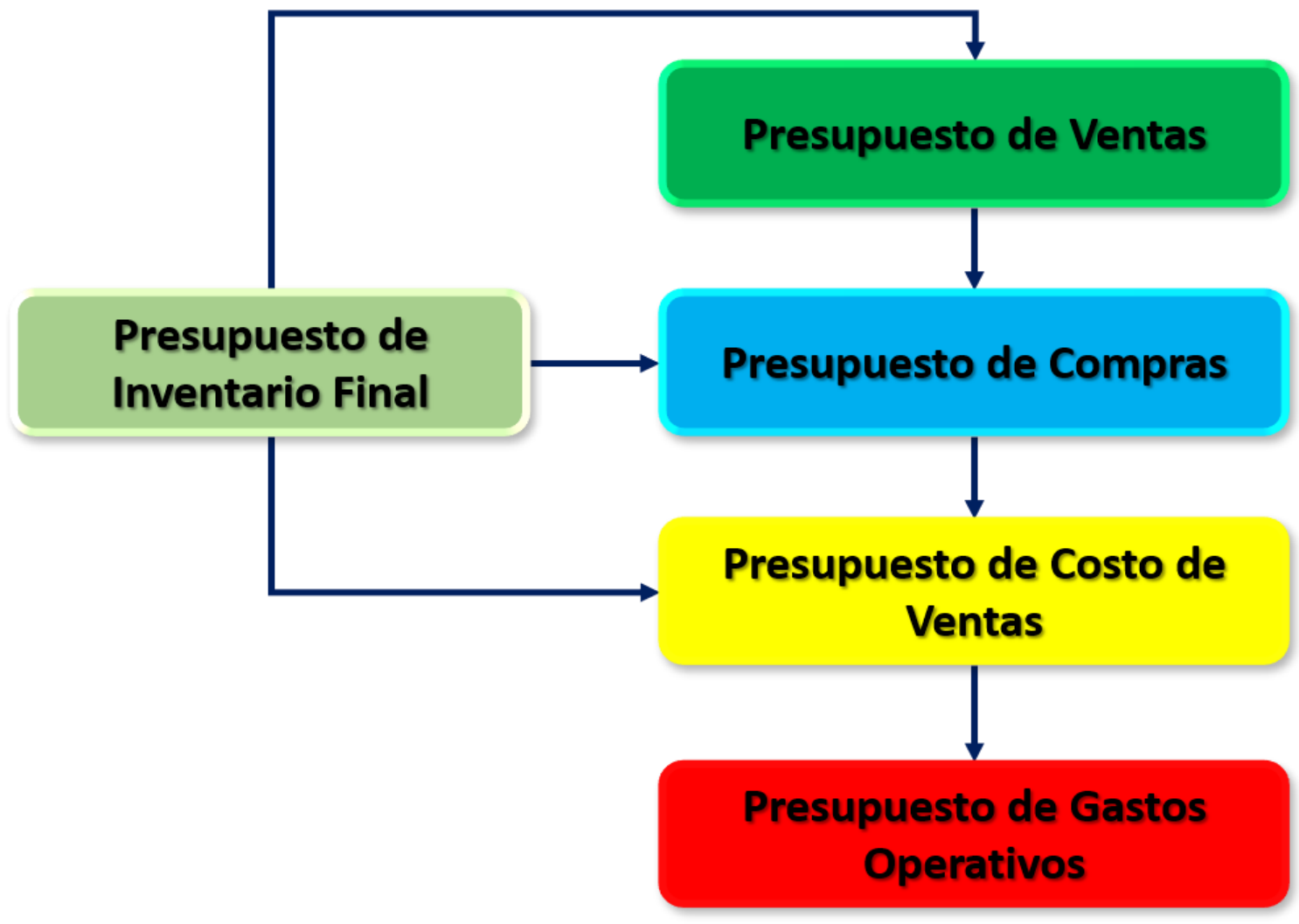

Nota: Se presentan los tipos de presupuestos organizacionales. Realizado en base a Horngren (2006).

\section{Control Interno}

Control Interno en la gestión la forma como se administran las empresas para enfrentar los riesgos de los negocios supone una gestión paralela de aseguramiento de actividades. Por tanto, lo pertinente es hablar de gestión de control y un mejor manejo de los recursos de forma que pueda afrontar sus responsabilidades futuras.

El control interno es una función fundamental de la gestión, aplicar herramientas que aporten una eficiente gestión de los procesos, será generadora de aseguramiento de las actividades operativas, minimizará los riesgos y permitir el logro de los objetivos planeados. 
REVISTA FAECO SAPIENS

\section{ISSN L 2644-3821}

Acceso Abierto. Disponible en:

https://revistas.up.ac.pa/index.php/faeco sapiens

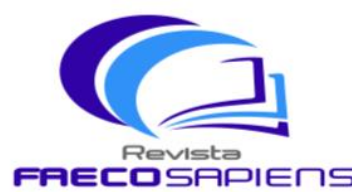

Volumen 4 - Número 1

enero-junio 2021

Recibido: $12 / 08 / 20$ Aceptado: $29 / 10 / 20$ pp. $44-57$

Indexada

Las empresas en su conjunto son responsables de aplicar el control de manera apropiada mediante la puesta en marcha de instrumentos de gestión, que permita el desarrollo concatenado de actividades y controles en la conducción hacia el logro de los objetivos comunes y mitigación de los riesgos.

Dextre y Del Pozo (2012), argumental que sobre la gestión y el control son:

La organización, a cargo de las actividades y su ejecución en una entidad, se define como el conjunto de personas comprometidas en la consecución de objetivos comunes mediante la ejecución de acciones debidamente coordinadas y comunicadas. Esta definición simple es muy compleja en la práctica, pues implica considerar características de los bienes y servicios a producir: ubicación, dimensión, extensión, entre otros factores, que determinan la naturaleza del giro del negocio, y condicionan el tipo y estructura de organización a instituir. Para ejecutar las actividades, la organización define las funciones que necesita y los sistemas y procedimientos con los cuales llevarlas a cabo y cumplir su cometido. Al ser la organización responsable de las actividades, lo es también del logro de los objetivos.

Las actividades, llevadas a cabo conforme han sido definidas e identificadas, son competencia de la organización. Es aquí donde aparece el control como la función de la administración que busca asegurar que las actividades se desenvuelvan tal y como han sido establecidas para cumplir los propósitos de la entidad en el logro de las metas y objetivos planeados. Puesto que el control asume la función de aseguramiento de actividades, ambos se encuentran íntimamente ligados en su operatividad.

En efecto, las actividades, al estar vinculadas a riesgos de no cumplimiento o la posibilidad de errores o desviaciones en su ejecución, tienen en el control la mitigación de esos riesgos mediante acciones de prevención, detección o corrección.

De esa manera, permiten la continuidad efectiva de los procesos y, sirven además en la evaluación y medición de los resultados de las decisiones tomadas con relación al modelo de negocio elegido. (pp. 71-72)

En tal sentido, queda claro que el control es la función que actúa sobre:

Los objetivos y métodos para comprobar su consistencia y corregir las desviaciones.

Las utilidades de las empresas en la consecución de los objetivos.

La gestión, en la ejecución de los procesos, para determinar si son apropiados.

El control se establece relacionado al esquema de la estructura de organización que adopta una empresa y requiere cubrir cuando menos los siguientes pasos:

Establecer normas y métodos para describir y ejecutar las actividades con los controles que las aseguren, así como las formas de medir el rendimiento

Comprobar si las actividades y los resultados corresponden al diseño y objetivos establecidos

Medir y evaluar los resultados en función de las metas alcanzadas. 
REVISTA FAECO SAPIENS

ISSN L 2644-3821

Acceso Abierto. Disponible en:

https://revistas.up.ac.pa/index.php/faeco sapiens
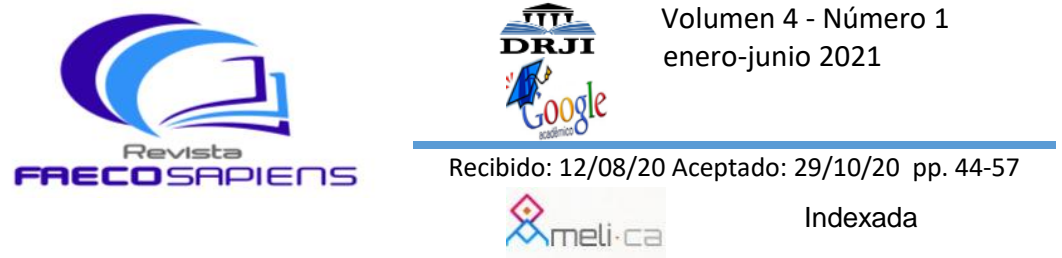

Tomar las acciones correctivas necesarias, verificando su cumplimiento

Efectuar los ajustes necesarios y las medidas que aseguren la gestión

Cruz (2013) en su trabajo expuesto en el Foro Miraflor, indica la importancia del control interno, donde dice que:

El control interno es todo un sistema de controles financieros utilizados por las empresas, y además, lo establece la dirección o gerencia para que estas puedan realizar sus procesos administrativos de manera secuencial y ordenada, con el fin de proteger sus activos, salvaguardarlos y asegurarlos en la medida posible, la exactitud y la veracidad de sus registros contables; sirviendo a su vez de marco de referencia o patrón de comportamiento para que las operaciones y actividades en las diferentes áreas de las empresas fluyan con mayor facilidad. (p. 26)

En la búsqueda de evidencia, deja expuesto la importancia del control interno en todas las estructuras de la empresa, en consecuencia, es fundamental utilizar herramientas como método de control interno para una mejor gestión en áreas específicas. Dejando en evidencia, que la implementación de un flujo de efectivo proyectado es de suma importancia para las cuentas por pagar, ya que provisiona a las empresas para hacerle frente a las responsabilidades con sus proveedores. Además, ayuda a la misma a crear y mantener la imagen necesaria, fortaleciendo la confianza con sus proveedores, para continuar manteniendo las relaciones comerciales de manera íntegra.

\section{Diseño de la Investigación}

Abreu (2012) expone que:

La investigación descriptiva encaja en las dos definiciones de las metodologías de investigación, cuantitativas y cualitativas, incluso dentro del mismo estudio. La investigación descriptiva se refiere al tipo de pregunta de investigación, diseño y análisis de datos que se aplica a un tema determinado. La estadística descriptiva responde a las preguntas quien, que, cuando, donde y como.

La estadística descriptiva utiliza técnicas de recolección de datos y análisis que produce informes relativos a las medidas de tendencia central, variación y correlación. La combinación de su resumen de características y estadísticas correlacionales, junto con su enfoque en los tipos específicos de preguntas de investigación, métodos y resultados es lo que distingue a la investigación descriptiva de los demás tipos de investigación. (p. 192-193)

Cazau (2006, citado por Abreu 2012) expone que la investigación correlacional presenta como "objetivo medir la relación que existe entre dos o más variables, en un contexto dado. Intenta determinar si hay una correlación, el tipo de correlación y su grado o intensidad. En otro sentido, la investigación correlacional busca determinar cómo se relacionan los diversos fenómenos de estudio entre sí". (p. 194) 
REVISTA FAECO SAPIENS

ISSN L 2644-3821

Acceso Abierto. Disponible en:

https://revistas.up.ac.pa/index.php/faeco sapiens
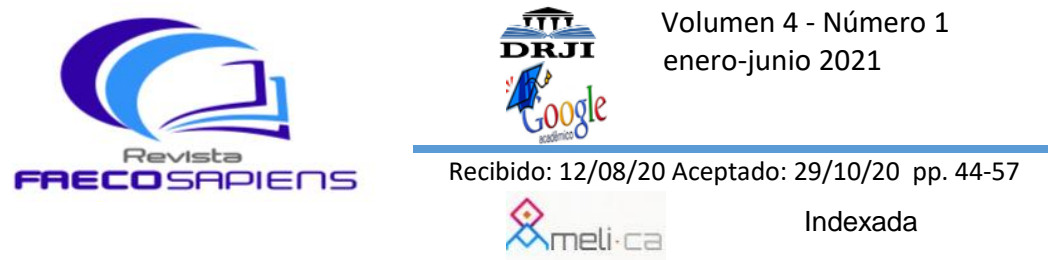

\section{Población y Muestra}

Expuesto lo anterior el presente estudio de acuerdo con la naturaleza del de la investigación, reúne las características de un estudio Descriptivo Correlacional, ya que abordará a un grupo de micros y pequeños empresarios para recabar los datos y poder demostrar como el flujo de efectivo proyectado, es una herramienta eficiente de control interno para que estas empresas logren resultado eficiente en las finanzas y la gestión de su modelo de negocio y puedan mantener el mismo en marcha

La población, muestra y muestreo son de importancia en la investigación para poder recabar la información y que esta apoye a demostrar los objetivos de esta para lo cual López define que:

Población: Es el conjunto de personas u objetos de los que se desea conocer algo en una investigación.

Muestra: Es un subconjunto o parte del universo o población en que se llevará a cabo la investigación.

Muestreo: Es el método utilizado para seleccionar a los componentes de la muestra del total de la población. (2004)

\section{Población}

Como se abordó en el Apartado II Tabla ,1 se pudo apreciar que para el año 2016 siendo la estadística más reciente de cual se tenga acceso a la información, se pudo percatar que en la República de Panamá se cuenta con un total de 43,328 microempresas y 6,689 medianas empresas en al Tabla 2, se puede observar el 45\%, que representa a la provincia de Panamá teniendo un total de 19498 para las micro y 3,010 para las medianas empresa de las cuales un $37 \%$ de esas empresas se ubican en la ciudad de Panamá siendo un total de 8,328 .

\section{Tabla 2.}

Población de Micro y Pequeñas Empresas en la Ciudad de Panamá.

\begin{tabular}{|l|r|r|}
\hline & Micro & Pequeñas \\
\hline Empresas en la Provincia de Panamá 45\% & 19,498 & 3,010 \\
\hline Total, Empresas en la Ciudad de Panamá 37\% & 7,214 & 1,114 \\
\hline Total & \multicolumn{2}{|c|}{$\mathbf{8 , 3 2 8}$} \\
\hline
\end{tabular}

Nota: Realización propia en base la información de la Tabla 1. 
REVISTA FAECO SAPIENS

ISSN L 2644-3821

Acceso Abierto. Disponible en:

https://revistas.up.ac.pa/index.php/faeco sapiens
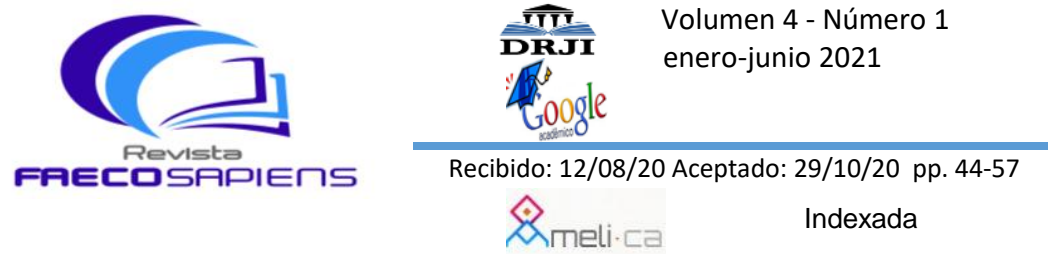

\section{Muestra}

En el muestreo, muestra puede ser obtenida de dos tipos: probabilística y no probabilística. "Las técnicas de muestreo probabilísticas permiten conocer la probabilidad que cada individuo a estudio tiene de ser incluido en la muestra a través de una selección al azar" (Otzen \& Carlos, 2017, p. 228).

En el presente estudio se utilizó el muestreo probabilístico simple donde todas tendrán la oportunidad de ser seleccionado, el mismo es de vital importancia, ya que se adapta a la investigación con una población de 8,328 micro y pequeñas empresas, en donde el nivel de confianza será de $95 \%$, con un margen de error $5 \%$, lo que arroja una muestra de 368 sujetos de estudio. Con respecto al cálculo de la muestra para poblaciones finitas mediante la fórmula $n=\frac{N \cdot Z^{2} \cdot p \cdot(1-p)}{(N-1) \cdot e^{2}+Z^{2} \cdot p(1-p)}$, se utilizó un método probabilístico, en donde se consideró un nivel de confianza $(Z)$ de $1.96 \%$, un porcentaje de error (e) de $0.05 \%$ y un valor proporción $(p)$ del $0.5 \%$, dando como resultado un tamaño de la muestra de 368 sujetos de estudio (Véase el procedimiento correspondiente en la siguiente Tabla 3).

\section{Tabla 3}

\section{Cálculo del Tamaño de la Muestra}

\begin{tabular}{l|l|l}
\hline Paso & \multicolumn{1}{|c}{ Actividad } & \\
\hline Paso 1. & Anota el tamaño de la población. & $\mathrm{N}=-8,328$ \\
Paso 2 & Selecciona un nivel de confianza. & \\
& Nivel de confianza & $\mathrm{Z}=95 \%$ \\
& Valor de Z & $\mathrm{Z}=1.96$ \\
Paso 3 & Porcentaje de error deseado (no mayor a 0.20). & $\mathrm{e}=0.05$ \\
Paso 4. & Valor de la proporción con la que deseas trabajar & $\mathrm{p}=0.5$ \\
Paso 5. & Identifica el tamaño de muestra & $\mathrm{n}=368$ \\
\hline
\end{tabular}

Nota: Elaboración propia en base a la utilización de la fórmula.

\section{Técnica e Instrumento de la Investigación}

Ruiz, las técnicas son recursos o procedimientos de los que se vale el investigador para acercarse a los hechos y acceder a su conocimiento y se apoyan en instrumentos para guardar la información tales como: el cuaderno de notas para el registro de observación y hechos, el diario de campo," entre otros elementos estrictamente indispensables para registrar lo observado durante el proceso de investigación (2011).

Rodríguez (2008, citado por Ruiz, 2011) expone que "las técnicas, son los medios empleados para recolectar información, entre las que destacan la observación, cuestionario, entrevistas, encuestas" (p. 169).

El presente estudio utiliza como técnica la encuesta siendo ampliamente utilizada en las investigaciones, ya que permite obtener y analizar los datos de modo rápido y eficaz. 
REVISTA FAECO SAPIENS

\section{ISSN L 2644-3821}

Acceso Abierto. Disponible en:

https://revistas.up.ac.pa/index.php/faeco sapiens

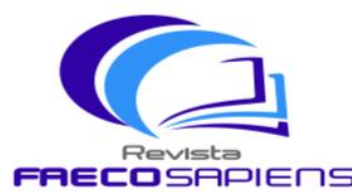

Volumen 4 - Número 1

enero-junio 2021

Recibido: $12 / 08 / 20$ Aceptado: 29/10/20 pp. 44-57

Indexada

Casas, et al., (2003) definen la encuesta como "una técnica que utiliza un conjunto de procedimientos estandarizados de investigación mediante los cuales se recoge y analiza una serie de datos de una muestra de casos representativa de una población o universo más amplio, del que se pretende explorar, describir, predecir y/o explicar una serie de características" (p. 527).

\section{CONCLUSIONES}

Para lograr un eficiente control interno las micros y pequeñas empresas deben contemplar las normas, políticas y procesos mínimos para poder lograr los resultados esperados, para tal fin toda empresa debe incorporar mejorar y dar cumplimiento a las normas establecidas.

Dotar a los empresarios de esta herramienta, les dará una ventaja competitiva en el mercado, ya que podrá contar con una gestión eficientes de los recursos, pronosticando las ventas y contar con el efectivo para la expansión de las ventas, cumplir con los compromiso, creando una buena imagen a la empresa, lo que conlleva a contar con financiamiento extra para crecer y expandirse, diversificando el negocio adaptándose a las nuevas tendencias y alargando el ciclo de vida, cumpliendo con los objetivos previos y el logro de las metas de sus dueños.

De acuerdo con la naturaleza del estudio de la investigación, reúne las características de un estudio Descriptivo, ya que abordará a un grupo de micros y pequeños empresarios para recabar los datos y poder comparar el flujo de efectivo proyectado, y si el mismo es una herramienta eficiente de control interno para que estas empresas logren resultado eficiente en la gestión de su modelo de negocio y puedan mantener el negocio en marcha

El resultado del estudio sustenta una propuesta metodológica de descripciones situacionales que impactará en los campos social, educativo y empresarial, ya que busca dotar a las micros y pequeñas empresas de una herramienta sencilla y fácil de realizar una mejor gestión de las finanzas.

Los micros y pequeños empresarios como nuevos emprendedores podrán contar con una guía práctica y didáctica de cómo implementar en sus negocios una herramienta la cual le brindará una forma de gestionar el efectivo, de manera tal que puedan pronosticar el margen de venta que desean obtener, así como los gastos que incurrirán, la forma de hacerle frente a las responsabilidades, las necesidades de financiamiento, lo mínimo que deben mantener y lo resultados que obtendrán en un periodo determinado.

Todas las micros y pequeñas empresas que podrán tener acceso a esta información y ponerla en práctica podrán ver los beneficios mencionado y poder tomar decisiones cónsonas a la realidad de su modelo de negocio y mantener el negocio en marcha, contar con prevención para hacerle frente al mercado cambiante y adaptarse a las necesidades del él, a la vez que puedan innovar en la búsqueda de expansión, innovación y supervivencia del negocio. 

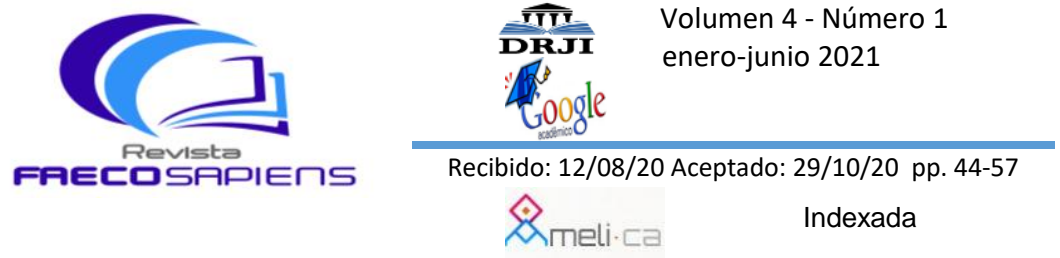

\section{REFERENCIAS BIBLIOGRÁFICAS}

Abreu, J. (Julio de 2012). Hipótesis, Método \& Diseño de Investigación. Daena: International Journal of Good Conscience, 187-197. Obtenido de https://n9.cl/0jcq

Autoridad de la Micro, Pequeña y Mediana Empresa. (2017). Informe: "Evolución y Estado de las Mipyme y el Emprendimiento". Panamá. Obtenido de https://n9.cl/fww0f

Casas Anguitaa, J., Repullo Labradora, J., \& Donado Campos, J. (2003). La Encuesta como Técnica de Investigación. Elaboración de Cuestionarios y tratamiento Estadístico de los Datos. Aten Primaria, 31(8), 527-538. Obtenido de https://n9.cl/su32

Castillero Mimenza, O. (20). Los 15 tipos de investigación (y características: La ciencia utiliza diferentes aproximaciones y técnicas para generar conocimiento acerca del mundo. Obtenido de Psicologia y Mente: https://n9.cl/5o2z

Cruz Cruz, L. M. (2013). Evaluación del control interno y su incidencia en la cuentas por cobrar de Foro Miraflor . Revista Científica de FAREM-Estel, 26-33.

Dextre Flores, J. C. (2012). ¿Control de gestión o gestión de control? Contabilidad y Negocios, vol. 7 , núm. 14, 2012,, 69-80.

López, P. (2004). Población, Muestra y Muestreo. Punto y Cero, 9(8), 69-74. Obtenido de https://n9.cl/qs80

Mera Portilla, M. A. (2017). Estado de flujo de efectivo en términos reales de la empresa Michell y Cía. SA - Lima 2011 - 2015. Universidad César Vallejo, Peru.

Otzen, T., \& Carlos, M. (2017). Técnicas de Muestreo sobre una Población a Estudio. Scielo, 227232. Recuperado el 2020, de https://n9.cl/pn0s

Rojas Villa, M. F. (2016). Interpretación del Estado de Flujo de Efectivo del Banco Central del Ecuador Durante los años 2010 - 2013 Mediante Análisis de Componentes Principales. Universidad del Azulay. María Fernanda Rojas Villa, Cuenca, Ecuador.

Ruiz Medina, M. I. (2011). Politicas Públicas en Salud y su Impacto en el Seguro Popular en Culiacán, Sinaloa, México. (Tesis Doctoral). Universidad Autónoma de Sinaloa, Sinaloa, Sinaloa, México. Obtenido de https://n9.cl/8sna

Serrano, N. V. (2016). Utilidad del Estado de Flujos de Efectivo en la detección de la manipulación contable. (Tesis Doctoral). Universitat Politècnica de Catalunya, Cataluña, España.

Solo Contabilidad. (22 de Agosto de 2019). Flujo de efectivo proyectado. Obtenido de https://n9.cl/bpiv

Toledo López, C. S. (2016). Elaboración, Análisis E Interpretación Del Estado De Flujos De Efectivo De Una Droguería. (Tesis). Universidad De San Carlos De Guatemala, Guatemala, Guatemala.

Zorrilla Melchor, J. H. (2015). El Estado de Flujos de Efectivo como estrategia Para la eficiente administración de los fondos en Las empresas comerciales de la provincia de Huaura. 